ISBN 978-981-14-1684-2

Proceedings of 2019 the 9th International Workshop on Computer Science and Engineering

(WCSE 2019 SUMMER)

Hong Kong, 15-17 June, 2019, pp. 213-218

doi: $10.18178 /$ wcse. 2019.06 .032

\title{
Design of Interactive System Based on Virtual Reality
}

\author{
Sun Yong ${ }^{1}$, Li Xinqi ${ }^{1+}$, Sun Miao $^{1}$ \\ 1 School of Automation and Electrical Engineering, Tianjin University of Technology and Education, Tianjin
}

\begin{abstract}
The interactive system based on virtual reality technology realizes data glove acquisition function through hardware circuits such as main control chip, bending sensor and MPU6050.Combining Kalman filter and arithmetic average filter, KNN algorithm extracts and processes the data glove information, and finally realizes the virtual interaction function successfully. The system has the characteristics of low cost, simple operation and strong immersion, and has wide application value..
\end{abstract}

Keywords: Interactive system, Virtual reality, hardware circuits

\section{Introduction}

Virtual Reality (VR) is an interdisciplinary technology that integrates artificial intelligence, multimedia, computer simulation, digital image processing and other disciplines ${ }^{[1]}$. Its interactivity, imagination and immersion reflect the full interaction between human and computer.Users in real environment can interact with computers through environment, natural language and body movements in virtual reality environment, and get timely and effective feedback information such as voice, picture, action, etc ${ }^{[2]}$. Then they can produce the same feeling as the real environment, as if they were in the situation.VR technology provides a new concept, a new way, a new content and a new method of human-machine interaction, which makes the interaction between people and virtual environment more vivid, harmonious and natural ${ }^{[3-4]}$.

In this paper, a human-computer interaction system is designed, which combines data gloves with VR glasses. Using Trinusvr software, the computer screen is projected to the mobile phone and divided into two screens. Then the mobile phone is placed in the VR glasses.Users only need to wear gloves, connect the receiver to the computer, open Nutritious Flying Screen software on the mobile phone and computer, and use WIFI hot spot connection to enter the virtual world of sensory games.

\section{System Principle}

The hardware of the system consists of left and right hand gloves, bending sensor and other hardware circuits. The core content is data acquisition and processing. Data acquisition mainly relies on the sensor's hand movement attitude and angle to collect information, and then obtain hand motion data. The technical difficulty lies in the need to properly handle the transmission timing protocol between different sensors when reading different sensors. Data processing is to analyze and calculate the collected data, use filters to remove noise in the data, and then separate the data of different sensors by data separation, and then obtain the threedimensional attitude data of each sensor, combined with the curvature sensor. The measured curvature value ultimately results in the restoration of hand movements. The system is integrated with VR virtual technology, using data gloves and VR glasses to provide users with a very immersive gaming experience. The hardware system block diagram is shown in Figure 1.

The system data processing and analysis technology mainly uses gesture recognition algorithm, and its data processing flow chart is shown in Figure 2. At the beginning, the data detected by the sensor is first

Corresponding author. Tel.: + 13622133013;

E-mail address: 184523662@qq.com 
extracted, and then the noise reduction processing is performed by filtering, and then the processed signal is calibrated to distinguish the data signals transmitted by different sensors, and the sensor signals are simply screened, and the rejection is obviously not The correct data signal is then intercepted according to the transmission protocol. Finally, the feature vector extraction and surgical pre-classification are used to extract the real reaction gesture motion characteristics, and then the effect of gesture restoration is reached.

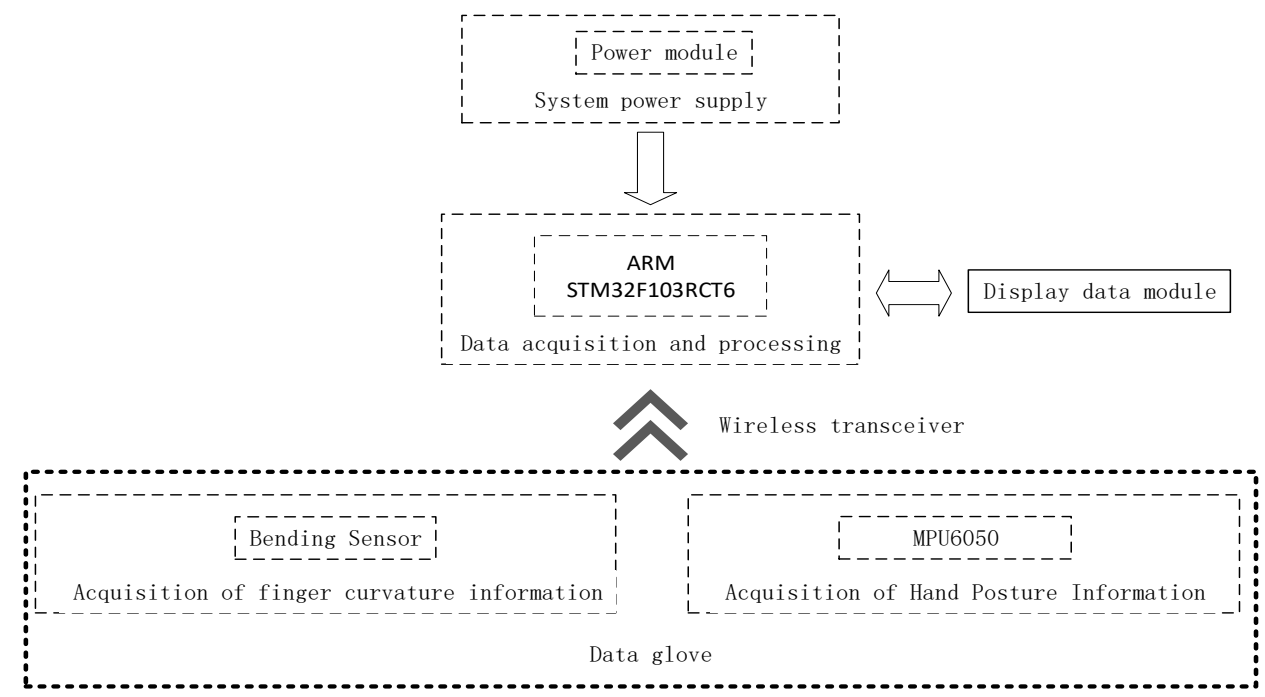

Fig. 1: System schematic block diagram.

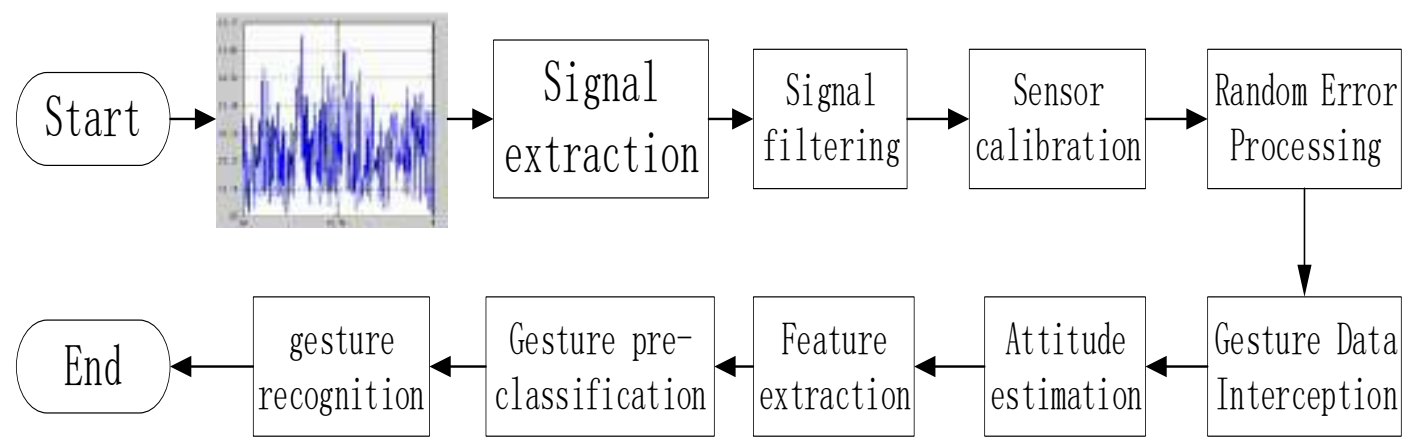

Fig. 2:Data processing flow chart.

\section{Design of Hardware System}

\subsection{Bending sensor}

The system's torsion sensor uses a flexible bending sensor to ensure free movement of the data glove. The bending sensor works by changing the resistance value when the sensor is bent to determine the degree of bending of the sensor. Its circuit schematic is shown in Figure 3. The on-resistance value of the bending sensor of the system under normal non-bending state is about $9 \mathrm{~K}$ ohms, and as the degree of bending increases, the resistance value also increases. The degree of bending of the sensor is linear with the value of the output resistance. When the degree of bending of the sensor reaches 90 degrees, the resistance value is about $14 \mathrm{~K}$ ohms; when it is bent 180 degrees, the resistance value is about $22 \mathrm{~K}$ ohms. The tested sensor can withstand more than 360 degrees of bending. The sensor can accurately reflect the angle of change of the finger, and has the characteristics of high sensitivity, low cost and convenient use. In this design, the resistor divider method is used to convert the change of the resistance value into the change of the voltage value, and then the output signal is amplified by the LM324 operational amplifier to obtain a relatively stable voltage, which facilitates the acquisition of data by the core chip ARM STM32F103RCT6. 


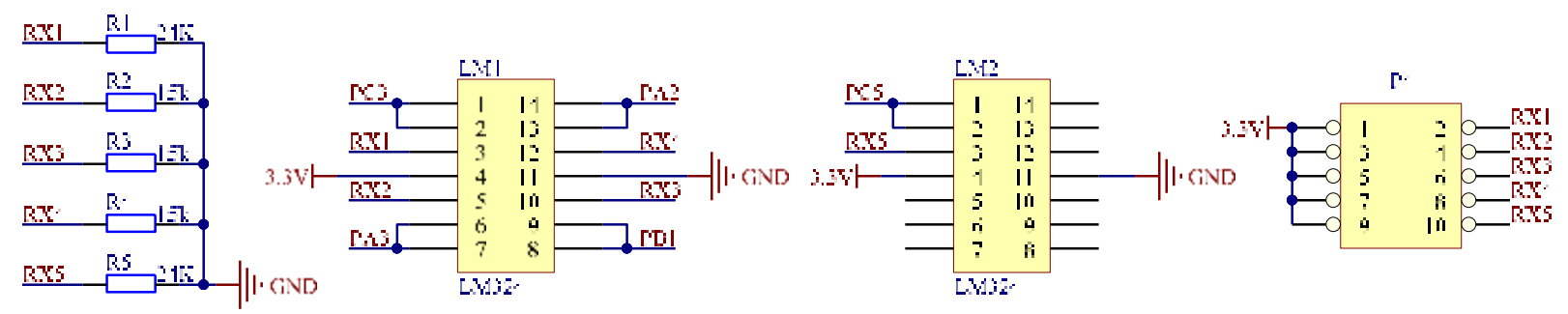

Fig. 3:Circuit schematic diagram of bending sensor

\subsection{NRF24L01 wireless transceiver module}

The nRF24L01 is a single-chip wireless transceiver chip manufactured by NORDIC that operates in the $2.4 \mathrm{GHz}$ to $2.5 \mathrm{GHz}$ ISM band. The schematic diagram of the NRF24L01 wireless transceiver module circuit is shown in Figure 4.

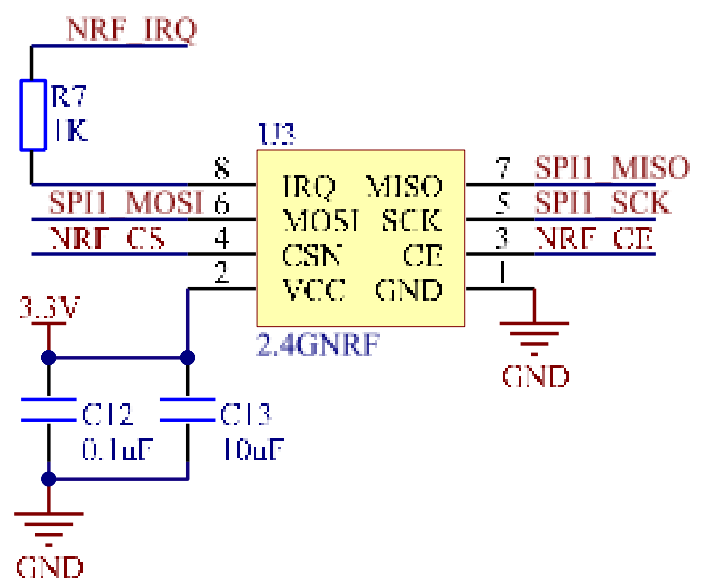

Fig. 4:Circuit schematic diagram of NRF24L01 wireless transceiver module

The wireless transceiver includes a crystal oscillator, a frequency generator, a power amplifier, an enhanced "SchockBurst" mode controller, a modulator, and a demodulation. The module output power channel selection and protocol settings can be set via the SPI interface. It has strong compatibility, can be connected to any micro controller chip, and complete wireless data transmission. When the module is running under the transmitter module, the current consumption is $11.3 \mathrm{~mA}$ when the transmit power is 0 $\mathrm{dBm}, 12.3 \mathrm{~mA}$ in the receive mode, and the current consumption is lower in the power-down mode and standby mode. The basic working principle is as follows: When the module needs to be in the transmit data state, firstly, the nRF24L01 needs to be configured into the transmit mode, and then the valid data TX_PLD and the receive node address TX_ADDR are written into the nRF24L01 buffer through the SPI port. Among them, TX_PLD must be continuously written when CSN is low, and TX_ADDR can be written once during transmission. Finally, CE is set to high level and kept for $10 \mu \mathrm{s}$, and the system delays $130 \mu \mathrm{s}$ to transmit data. When receiving data, it is also necessary to first The nRF24L01 is configured to receive the mode, and then sets the delay time to $130 \mu \mathrm{s}$ and then enters the receive wait state. At this time, the module is in the wait state and waits for the received data. When the module detects a valid data address and CRC, the system stores the packet data in the RX FIFO and sets the interrupt flag high to generate an interrupt, storing the received data in the MCU.

\subsection{Display Circuit}

The system display selects YAOXY12864B Chinese LCD dot matrix display, and its system schematic is shown in Figure 5. YAOXY12864B is a new type of LED touch screen with 8-bit parallel or 3-wire serial interface. It integrates 8192 Chinese characters and 128 ASCII character sets. Compared with traditional touch screens, it has less information and a single form. Poor machine interaction, high operator requirements, this type of touch screen has low power consumption, small size, good interactivity and other excellent performance, in addition to the original single display characters can be added to display graphics, curves and other functions, and The function of realizing the dynamic effect of the screen and the left and 
right scrolling makes the human-machine interface more friendly and the operation is more flexible and convenient.



Fig. 5: Schematic diagram of display circuit.

\section{System Software Design}

\subsection{Arithmetic average filtering method}

In general, the data extraction is located at the lowest end of the gesture recognition system, and the purpose of the data extraction is to extract the original acceleration signal and the angular velocity signal related to the gesture. These raw and unprocessed signals are related to the recognition rate of the entire system, because the original acceleration signal and angular velocity signal are inevitably used in the system. When designing the system, it is necessary to identify the collected acceleration data and angular velocity data to an external storage unit. Therefore, the stability and integrity of the transmission of data will affect the accuracy of the identification. There are two general types of data transmission: wired transmission and wireless transmission. Considering the actual use of the project, a wireless transmission method is adopted. And the sampling frequency of the accelerometer and the gyroscope is 100HZ.

Because the curvature signal collected by the digital collector has unstable fluctuations, the system uses the arithmetic average filtering method for filtering. Continuously take the $\mathrm{N}$ sample values of the system for arithmetic average operation. When the $\mathrm{N}$ value is large: the signal smoothness is higher, but the sensitivity is lower; when the $\mathrm{N}$ value is smaller: the signal smoothness is lower, but the sensitivity is higher; the general flow rate, $\mathrm{N}=12$. When the signal has general random noise, the method is used to filter and obtain an average value, so that the signal fluctuates up and down within a certain range of values, thereby obtaining a smooth and stable value. The system programming code is as follows:

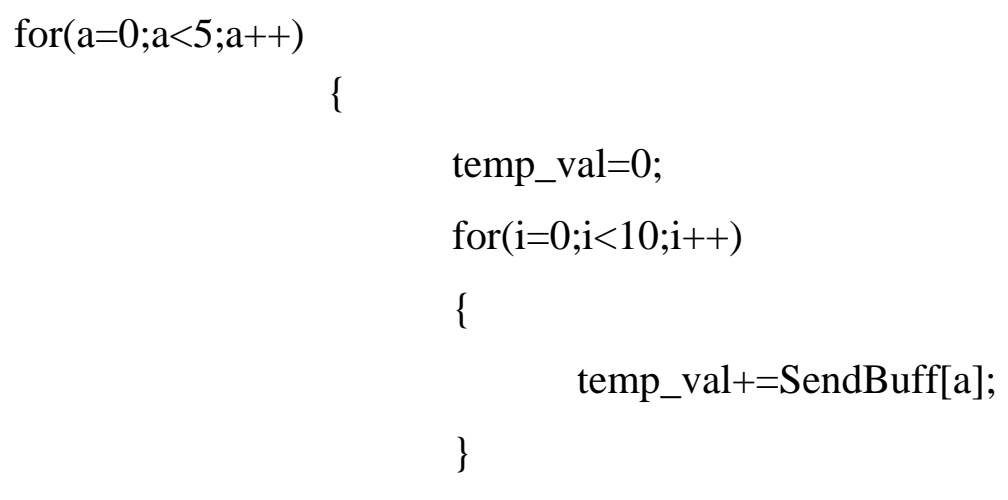

ADCmun[a]=temp_val/10; 


\section{2. k-nearest neighbor algorithm}

The KNN algorithm classifies by measuring the distance relationship between different eigenvalues. If a sample data has the $\mathrm{k}$ most similar in the feature space, that is, the nearest neighbor of the feature space belongs to a certain category, the sample also belongs to this category. . In general, $\mathrm{K}$ is an integer less than 20. The premise of the KNN algorithm is that the selected neighbors are all objects that have been classified, and only the nearest sample data is used in the decision to determine the category to which the sample belongs. The schematic diagram is shown in Figure 6.

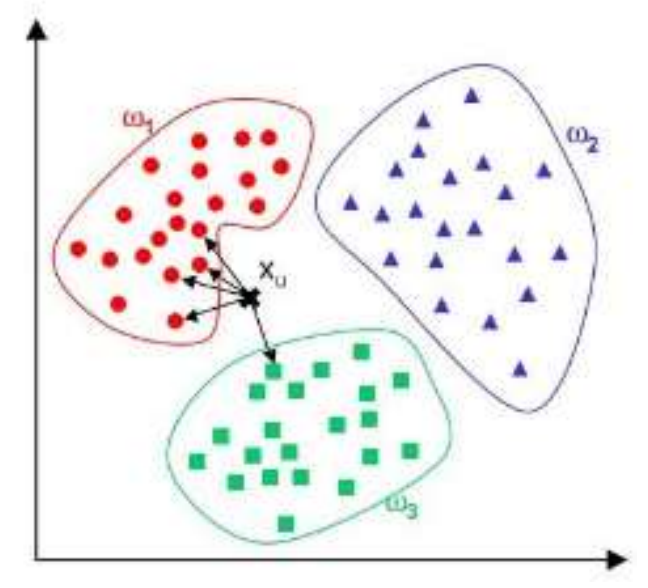

Fig. 6:KNN algorithm schematic diagram.

When inputting the training set, where is the n-dimensional instance feature vector, the instance class, the instance $\mathrm{x}$ is predicted, and the output $\mathrm{x}$ belongs to the class $\mathrm{y}$. The distance is determined by using the Euclidean distance and $\mathrm{k}$ sample points close to $\mathrm{x}$ are found in the training set, and finally the category to which $\mathrm{x}$ belongs is determined according to the majority voting principle.

\section{Results and Analysis}

After a series of debugging experiments, the system successfully realized the combination of the real motion and the virtual world, replacing the mouse and keyboard with gloves and user movement changes, and performing synchronization with the game action, combined with the use of VR glasses, enhanced Visual effects. The combination of the two makes the game not only implemented in the display. After wearing VR glasses, a full-screen game will appear in the line of sight, and then the action of the game will be controlled by hand to make the immersion stronger. The actual physical test diagram of the system is shown in Figure 7 below.

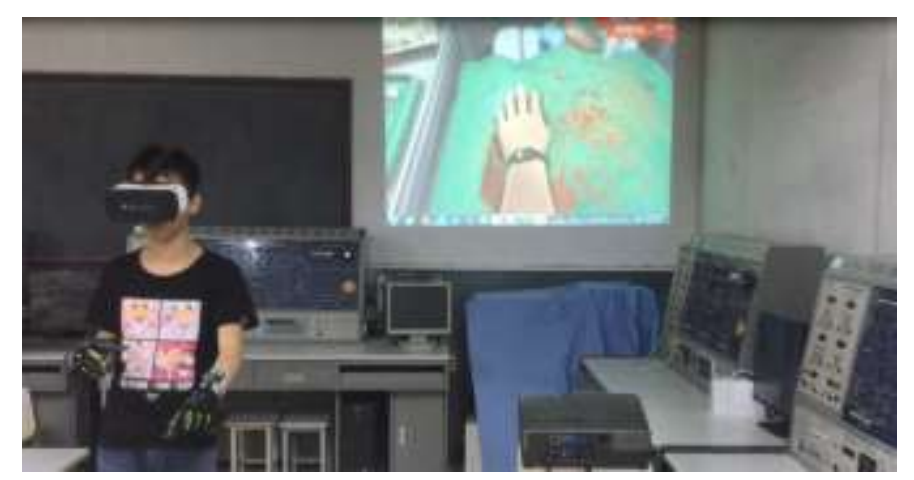

Fig. 7: The actual physical test diagram of the system

In summary, the system has stable operation, high reliability, low cost, simple operation and strong immersion, and has wide application value and promotion prospects. 


\section{Acknowledgments}

This work was supported by the National Key Research and Development Program of China (Grant No. 2017YFB0403802) and the Scientific Research Development Fund Project of Tianjin University of Technology and Education (KJ1923).

\section{References}

[1] Weiyin Zuo. Research on the Application of Virtual Reality Technology in Handicraft Display[A]. International Information and Engineering Association.Proceedings of 2018 International Conference on Data Processing,Artificial Intelligence, and Communications(DPAIC 2018)[C].International Information and Engineering Association:Computer Science and Electronic Technology International Society.2018:4.

[2] Huang Chengyun. Analysis on the Practice of Virtual Reality Technology in Electric Emergency Repair Practice[A]. International Information and Engineering Association.Proceedings of 2018 7th International Conference on Advanced Materials and Computer Science(ICAMCS 2018)[C].International Information and Engineering Association:Computer Science and Electronic Technology International Society,2018:6.

[3] Jing Su. Research on Application of Virtual Reality Technology in Production Practice of Bioengineering Specialty[A]. Hainan University, Sanya University, Xiamen University Tan Kah Kee College.Proceedings of 4th International Conference on Social Science and Higher Education(ICSSHE 2018)(Advances in Social Science,Education and Humanities Research,VOL.181)[C].,2018:3.

[4] Lei Xie. Research of Computer-aided Instruction based on Virtual Reality Technology[A]. Institute of Management Science and Industrial Engineering.Proceedings of 2018 3rd International Conference on Education \&amp; Education Research(EDUER 2018)[C].Institute of Management Science and Industrial Engineering:Computer Science and Electronic Technology International Society,2018:5. 